\title{
Tier-scalable reconnaissance: the future in autonomous C4ISR systems has arrived: progress towards an outdoor testbed
}

Wolfgang Fink, Alexander J.-W. Brooks, Mark A. Tarbell, James M. Dohm 


\title{
Tier-Scalable Reconnaissance ${ }^{T M}$ : The Future in Autonomous $C^{4}$ ISR Systems has arrived Progress towards an Outdoor Testbed
}

Wolfgang Fink*a , Alexander J.-W. Brooks ${ }^{\mathrm{a}}$, Mark A. Tarbell ${ }^{\mathrm{a}}$, James M. Dohm ${ }^{\mathrm{b}}$

${ }^{a}$ Visual and Autonomous Exploration Systems Research Laboratory, College of Engineering, University of Arizona, 1230 E Speedway Blvd, Tucson, AZ 85721, USA; ${ }^{b}$ Foundation for Advancement of International Science, 24-16, Kasuga, 3-chome, Tsukuba, Ibaraki 305-0821, Japan

In Memoriam Lawrence "Larry" E. Gilbert (1932 - 2016) of Caltech

\begin{abstract}
Autonomous reconnaissance missions are called for in extreme environments, as well as in potentially hazardous (e.g., the theatre, disaster-stricken areas, etc.) or inaccessible operational areas (e.g., planetary surfaces, space). Such future missions will require increasing degrees of operational autonomy, especially when following up on transient events. Operational autonomy encompasses: (1) Automatic characterization of operational areas from different vantages (i.e., spaceborne, airborne, surface, subsurface); (2) automatic sensor deployment and data gathering; (3) automatic feature extraction including anomaly detection and region-of-interest identification; (4) automatic target prediction and prioritization; (5) and subsequent automatic (re-)deployment and navigation of robotic agents. This paper reports on progress towards several aspects of autonomous $\mathrm{C}^{4}$ ISR systems, including: Caltech-patented and NASA award-winning multi-tiered mission paradigm, robotic platform development (air, ground, water-based), robotic behavior motifs as the building blocks for autonomous telecommanding, and autonomous decision making based on a Caltech-patented framework comprising sensor-data-fusion (feature-vectors), anomaly detection (clustering and principal component analysis), and target prioritization (hypothetical probing).
\end{abstract}

Keywords: Autonomous $\mathrm{C}^{4}$ ISR systems, smart service systems, multi-tiered architectures, robotic agents, navigational behavior motifs, sensor-data-fusion framework, objective anomaly detection, target prioritization

\section{INTRODUCTION TO THE TIER-SCALABLE RECONNAISSANCE PARADIGM}

NSF defines a "smart" service system as follows: "A system capable of learning, dynamic adaptation, and decision making based upon data received, transmitted, and/or processed to improve its response to a future situation. [...] These capabilities are the result of the incorporation of technologies for sensing, actuation, coordination, communication, control, etc. The system may exhibit a sequence of features such as detection, classification, and localization that lead to an outcome occurring within a reasonable time." [NSF PFI:BIC 2015]. This definition of a "smart" service system is synonymous to that of an autonomous system and sets the stage for the following.

Robotic reconnaissance/exploration agents are called for in extreme environments, e.g., planetary surfaces and space, as well as in potentially hazardous or inaccessible operational areas, such as mine fields, the theatre, enemy occupied territories, natural disaster-stricken areas, or areas that have been exposed to biochemical (warfare) agents or radiation. They are also necessary to identify, map, and characterize prime science targets, including those that transiently exist (e.g., volatile release such as water or methane), or those that cannot be examined in situ due to current mission and engineering constraints (e.g., rugged mountainous terrain) on Mars or other planetary bodies. For such missions, increasing degrees of operational autonomy are required, comprising the following requirements: (1) Automatic characterization of operational areas from different vantages, (2) automatic sensor deployment and data gathering, (3) automatic feature extraction, anomaly detection, and region-of-interest identification, (4) automatic target prioritization, and (5) subsequent automatic redeployment and navigation of robotic agents.

\footnotetext{
* e-mail: wfink@autonomy.caltech.edu; phone: +1-520-621-8734; website: http://autonomy.caltech.edu
} 
To that effect the Visual and Autonomous Exploration Systems Research Laboratory at Caltech (2003 - 2016) and at the University of Arizona (2009 - present) has devised a NASA award-winning (NASA Board Award 2009), widely publicized, ${ }^{1-15}$ and Caltech-patented ${ }^{16-18}$ mission paradigm, termed Tier-scalable Reconnaissance (TSR), as the foundation for autonomous $\mathrm{C}^{4}$ ISR systems of the future. Originally devised for autonomous robotic space exploration of planetary surfaces, the tier-scalable reconnaissance paradigm integrates multi-tier (space, atmosphere, surface, subsurface) and multi-agent hierarchical mission architectures (Fig. 1), introducing not only mission redundancy and safety, but also enabling intelligent, objective-driven, and distributed reconnaissance in real time.

NASA and ESA have already adopted the tier-scalable reconnaissance mission architecture paradigm. This is evidenced by the early use of Mars Reconnaissance Orbiter (MRO) with its HiRISE onboard camera system to image and help navigate the Mars Exploration Rovers Spirit and Opportunity from orbit. ESA's ExoMars mission, by design, will comprise a Mars orbiter, surface platform, and a Mars rover. Moreover, the NASA-ESA Titan Saturn System Mission (TSSM) has been considering a tier-scalable reconnaissance type integrated mission architecture for the exploration of Titan comprising a Titan orbiter, Montgolfière type hot-air balloon, and potential surface elements such as lake landers (e.g., Titan Mare Explorer (TiME)) for in-situ exploration of the hydrocarbon lakes on Titan. The Russian Venera-D mission is considering combinations of an orbiter, sub-satellite, two balloons at different altitudes, and VEGA-type lander, long living station, or mini probes dropped from the lower balloon - yet another example of a tier-scalable reconnaissance mission architecture.

Over the past several years the Visual and Autonomous Exploration Systems Research Laboratory has been leading multi-disciplinary and multi-institutional research and development efforts towards tier-scalable reconnaissance mission architectures, autonomous $\mathrm{C}^{4}$ ISR systems, autonomous robotic exploration and reasoning systems, as well as research on automated global feature analysis with particular focus on unbiased anomaly detection and target prioritization. With funding from NASA, DOE, and NSF the lab has been developing an outdoor-deployable robotic $\mathrm{C}^{4}$ ISR testbed for autonomous operations in aerial, terrestrial, and riverine/maritime environments to investigate how a system, through a sequence of events, such as detection (via sensors), classification (via cognitive capabilities), and localization, can arrive at a decision and course of action within a reasonable amount of time - an autonomous TSR system.

To demonstrate the capabilities and inherent abilities of TSR architectures as the foundation of $\mathrm{C}^{4}$ ISR systems of the future, we report in the following on progress towards the establishment of an outdoor testbed for TSR missions and/or $\mathrm{C}^{4}$ ISR operations.

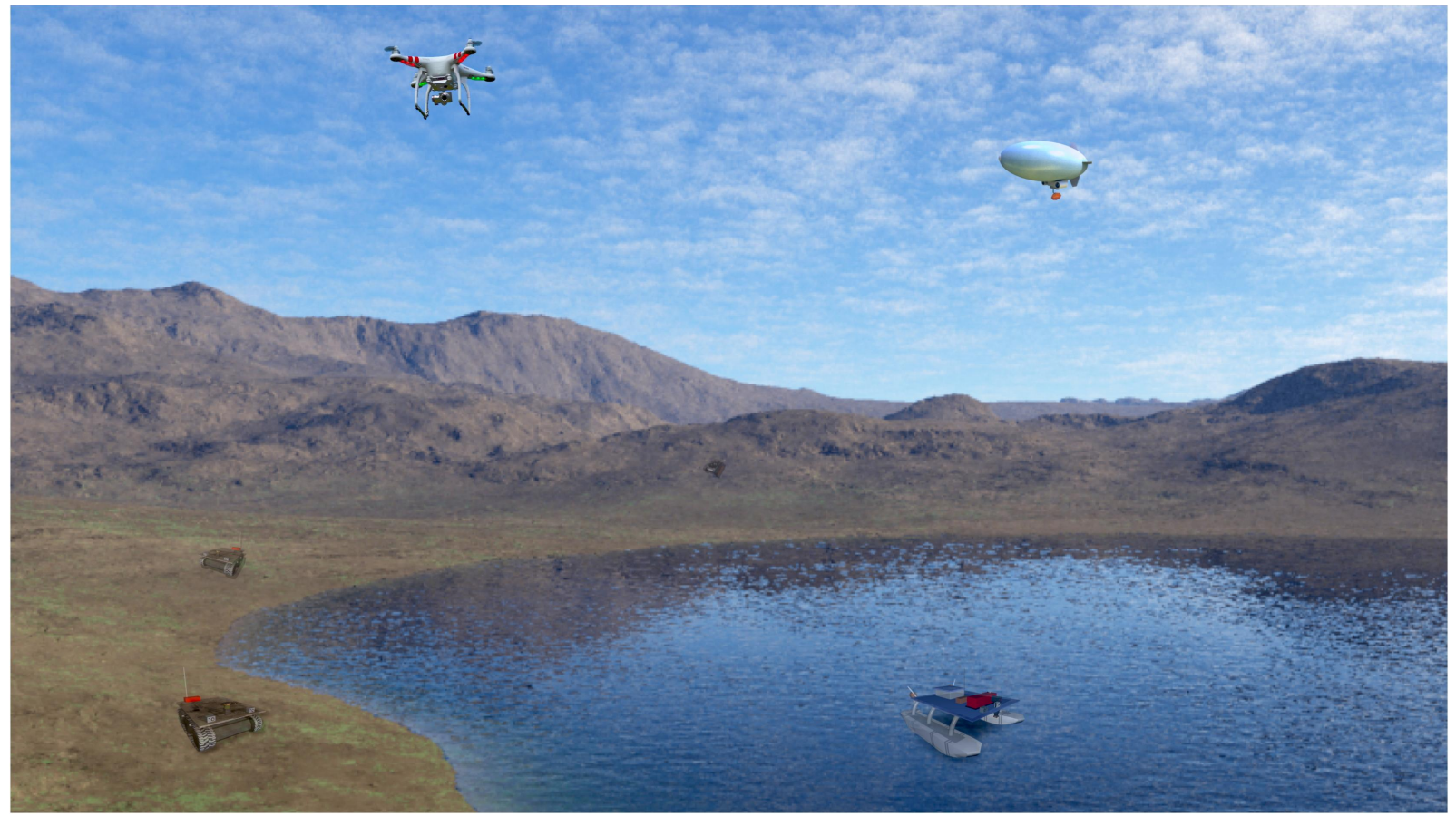

Figure 1. Typical deployment scenario of airborne agents (quadcopter and blimp), land-based agents (land rovers), and water/liquid-based rovers (sea rover). 


\section{DESCRIPTION OF CURRENT SET OF ROBOTIC AGENTS OF THE TESTBED}

The Visual and Autonomous Exploration Systems Research Laboratory (http://autonomy.arizona.edu) has developed a robotic multi-agent testbed that currently comprises computer-controlled (versus remote controlled) robotic ground surface vehicles ("Land Rovers"), robotic sea surface vehicles ("Sea Rovers"), and robotic unmanned aerial vehicles ("Air Rovers"). These vehicles can be equipped with sensors for exploration, environmental monitoring, and Department of Defense (DOD) relevant applications, such as reconnaissance, surveillance, subsurface exploration, cleanup operations, and generic asset delivery.

A typical TSR scenario begins by deploying overhead reconnaissance, utilizing air rovers, to obtain a generalized perspective of the operational area of interest (Fig. 1). Once targets of interest have been identified from the air, a second level of TSR comes into play, viz. ground-level reconnaissance, which is effected by ground-level deployment of land rovers (Fig. 1). The fleet of small, potentially expendable, purposed land rovers are equipped with specialized instrumentation appropriate for the tasks/research to be accomplished. After deployment by the overhead reconnaissance (or through independent deployment), the land rovers are being autonomously telecommanded by the overhead reconnaissance (Section 3) to investigate in-situ the targets of interest previously identified by the overhead reconnaissance, using, e.g., the Automated Global Feature Analyzer (AGFA, Section 4) and other methodologies. Additionally, should there be pools or bodies of water or other liquids (e.g., hydrocarbons such as on Saturn's moon Titan) requiring reconnaissance, sea rovers are deployed to explore those regions. Areas/targets of declared interest, as well as anomalous elements, are identified through the use of AGFA.

In the following we detail the specifications of the current types of robotic agents in the TSR outdoor testbed.

\subsection{Air Rovers}

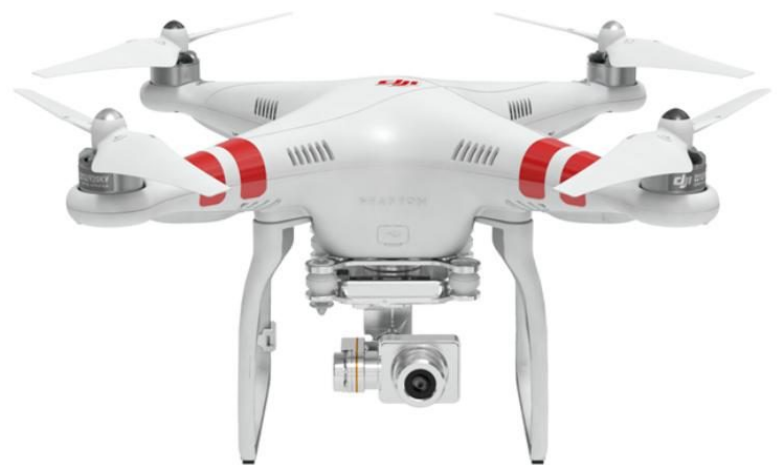

Figure 2. Air rover: here a quadcopter equipped with an onboard camera.

Specific attributes and capabilities of the robotic unmanned aerial vehicles:

- Chassis: Lightweight strengthened polycarbonate chassis and descending sensor platform.

- CPU: General-purpose, Raspberry Pi micro Unix workstation; Wireless Internet and GPS capability.

- Power Supply: High current (5 Ah), high voltage (15.2 V) LiPo 4S rechargeable power cells. Provides up to 25 minutes on one battery charge.

- Propeller Motors: 4 independently operated and controlled motors. Capable of flight reaching $6000 \mathrm{~m}$ altitude at $16 \mathrm{~m} / \mathrm{s}$ rate.

- Camera: $12 \mathrm{MP}$ CMOS, $4 \mathrm{k}$ x $3 \mathrm{k}$ stills, $2.7 \mathrm{k}$ x $1.5 \mathrm{k}$ video.

- Dimensions: Base 350mm (diagonal); $1.2 \mathrm{~kg}$ unladen.

- Sensor Platform: Configurable sensor platform capable of carrying up to $365 \mathrm{~g}$ of payload equipment, sensors, and instrumentation. 


\subsection{Land Rovers}
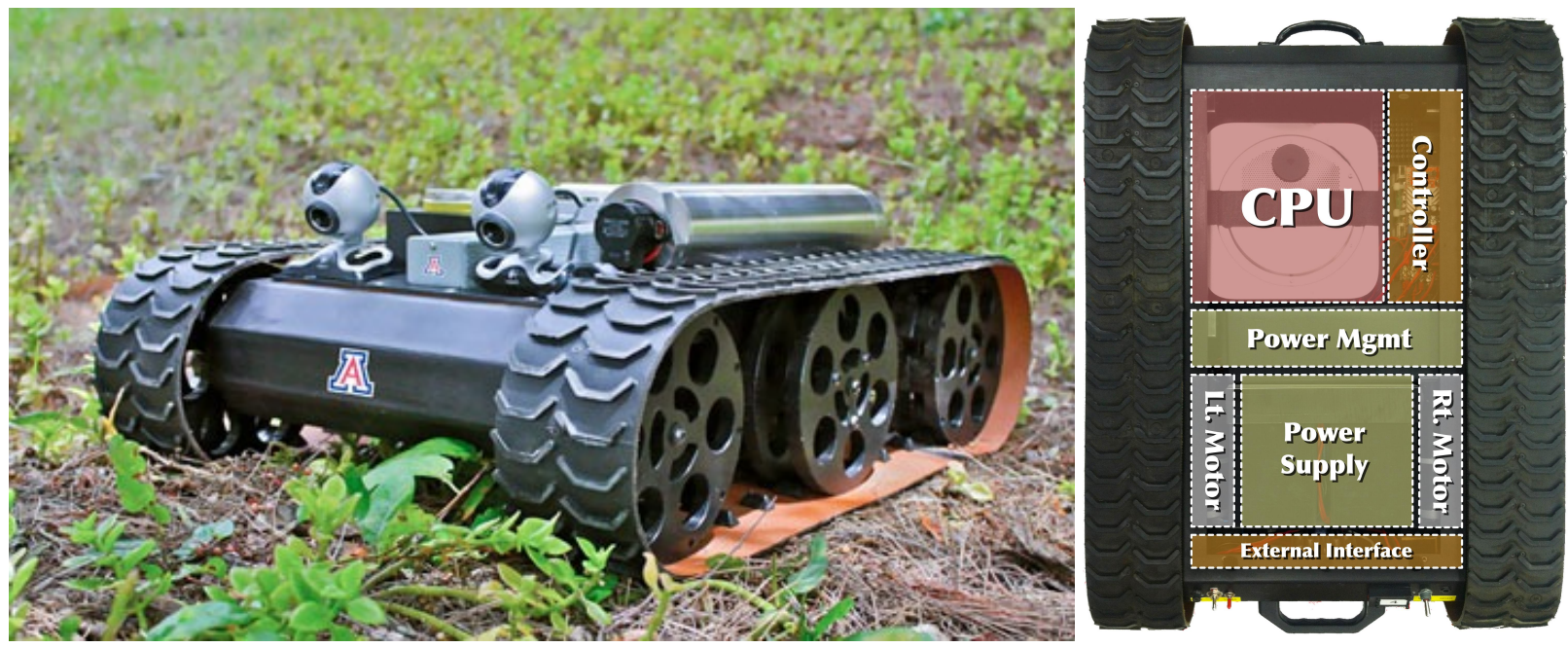

Figure 3. Land rover (left) and schematic of the interior functional units (right).

Specific attributes and capabilities of the robotic ground surface vehicles:

- Chassis: Ruggedized anodized aluminum unitized chassis and elevated sensor platform with dual-side treads, 6 tread wheels, 2 motors.

- CPU: General-purpose, high-performance (dual-core) Apple Mac mini Unix workstation; Wireless Internet capability.

- Controller: Dual-channel 35 A microprocessor-based motor controller with thermal and over current protection as well as quadrature encoder feedback capabilities.

- Power Management: High current power distribution and routing module.

- Power Supply: High current (15 Ah), high voltage $(24 \mathrm{~V})$ matched rechargeable power cells. Provides up to 10 hours / $10 \mathrm{~km}$ on one battery charge. Front panel rechargeable.

- Left \& Right Motors: Type $0452 \mathrm{~mm}$ diameter, steel gearhead 1:43 reduction $82 \mathrm{rpm}$ motors, with stall torque exceeding $300 \mathrm{kgf}-\mathrm{cm}$. 500 Counts per Revolution with 3 channel TTL level quadrature outputs giving signals for each revolution of the motor (index), allowing detection of direction and speed of motors. Typical travel speed $1 \mathrm{~m} / \mathrm{sec}$.

- External Interface: Electronics panel supporting various initiation switches, and battery recharging port.

- Lidar: Scanning Laser Rangefinder capable of reporting ranges from $150 \mathrm{~mm}$ to $30 \mathrm{~m}$ (1 mm resolution) in a $270^{\circ}$ arc $\left(0.25^{\circ}\right.$ angular resolution $)$, at a rate of 25 sweeps $/ \mathrm{sec}$.

- Dimensions: Base 27.5"L x 20"W x 9.5"H; sensor platform 21"L x 11.5"W; 40 lbs unladen.

- Sensor Platform: Swappable, configurable sensor platform capable of carrying up to $70 \mathrm{~kg}$ of payload equipment, sensors, and instrumentation. 


\subsection{Sea Rovers}

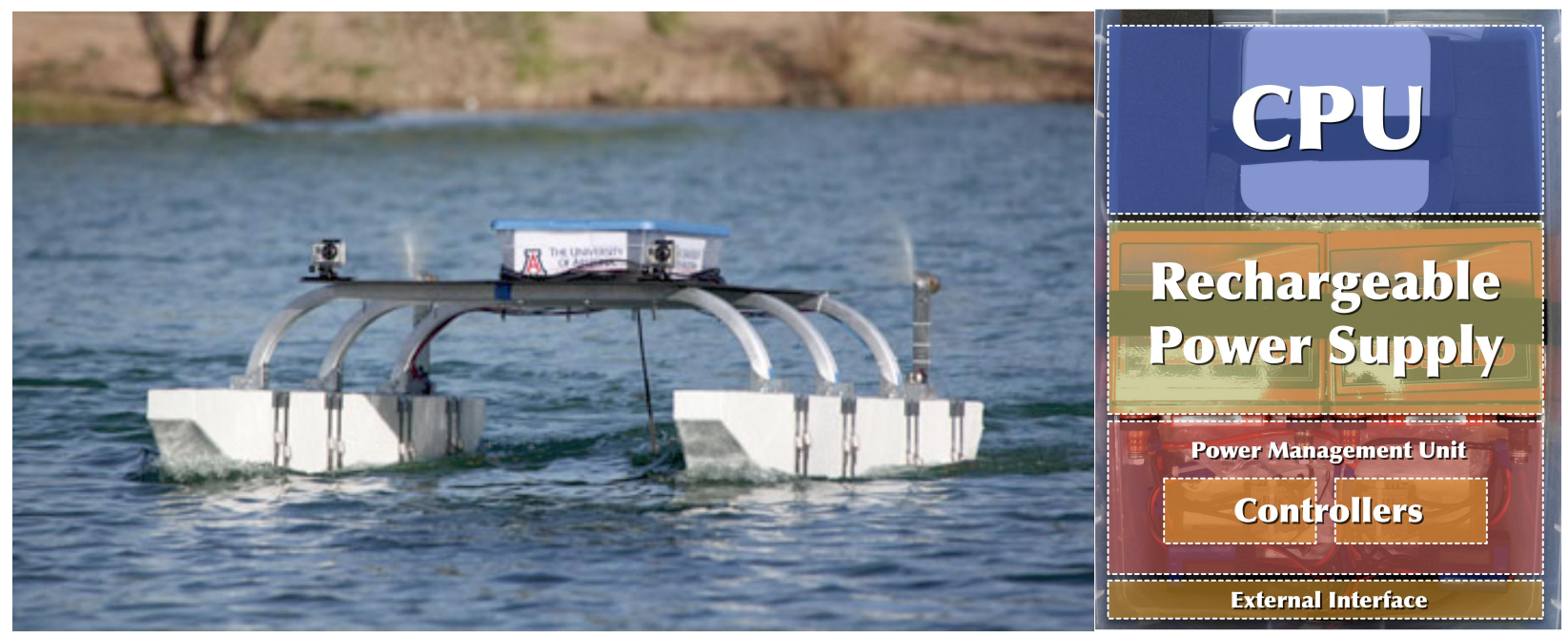

Figure 4. Sea rover deployed (left) and schematic of the interior functional units (right). Note the identical interior makeup as the land rover (Fig. 3).

Specific attributes and capabilities of the robotic sea surface vehicles:

- Chassis: Extruded arched aluminum chassis bars mounted atop two hardened, fortified pontoons, forming a catamaran-type structure, with multi-partitioned Delrin ${ }^{\circledR}$ (acetal homopolymer) sensor platform on top.

- CPU: General-purpose, high-performance (dual-core) Apple Mac mini Unix workstation; Wireless Internet capability.

- Controllers: Dual-channel 150 A microprocessor-based motor controllers with thermal and over current protection.

- Power Management: High current power distribution and routing module.

- Power Supply: Ultra high current (680 CCA), high voltage (24 V) dual rechargeable power cells. Provides up to 16 hours propeller power on one battery charge.

- Left \& Right Motors: Brushless type 14x10 CAM Carbon, 3-phase, direct-drive 10,000 rpm motors. $1 \mathrm{~kW}$ power output, $85 \%$ efficiency rating.

- External Interface: Electronics interfacing section supporting external device connection (Sonar, cameras, Lidar, etc.).

- Lidar: Scanning Laser Rangefinder capable of reporting ranges from $150 \mathrm{~mm}$ to $30 \mathrm{~m}$ (1 $\mathrm{mm}$ resolution) in a $270^{\circ}$ arc $\left(0.25^{\circ}\right.$ angular resolution $)$, at a rate of 25 sweeps $/ \mathrm{sec}$.

- Dimensions: Base 73"L x 58.5"W x 20"H; sensor platform 36"L x 46"W; 90 lbs unladen.

- Sensor Platform: Swappable, configurable sensor platform capable of carrying up to $70 \mathrm{~kg}$ of payload equipment, sensors, and instrumentation.

\section{ROBOTIC BEHAVIOR MOTIFS FOR AUTONOMOUS TELECOMMANDING}

The navigational behavior of the autonomous robotic surface agents (i.e., land and sea rovers), used for in-situ investigation of the targets of interest previously identified by the overhead reconnaissance, is the aggregation of several independent operational modules or motifs. These robotic behavior motifs analyze $270^{\circ}$ arc $2 \mathrm{D}$ Lidar sweeps and exhibit responses that can be compartmentalized and prioritized to intelligently handle complex scenarios. As a proof of 
concept, this paper showcases the core obstacle avoidance algorithm, as well as a continuous driving module that encapsulates it, in the following subsections.

\subsection{Obstacle Avoidance Motif}

Safety is the underlying priority of robotic agents in the tier-scalable autonomy paradigm. Consequently, obstacle avoidance is the most fundamental behavioral motif and operates underneath all other modules. When a potential collision is detected, any navigational algorithms are overridden by protocols designed to protect the agent. It follows that for the standalone obstacle avoidance behavior, the agent resides in an idle state until an obstacle comes too close to it. When approached, the robotic platform will verify that it has space to center the obstacle (making its own if necessary), pivot until the obstacle is centered, and retreat to a safe distance (Fig. 5).

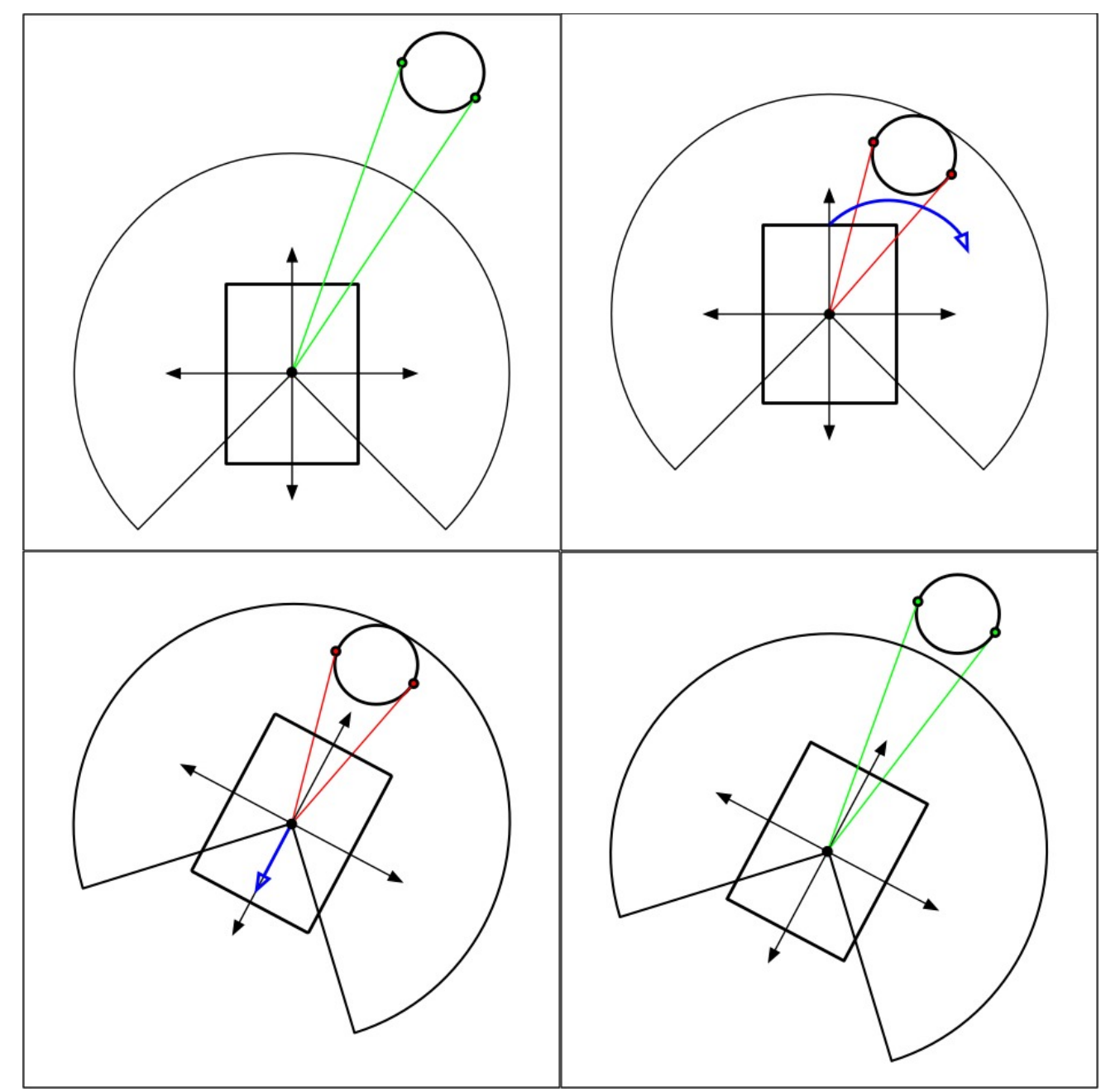

Figure 5. Upper Left: agent is in its idle state since all obstacles are a safe distance away. Upper Right: agent starts pivoting to center an obstacle threatening a collision ahead. Bottom Left: obstacle is centered and the agent begins to back away to create a safe space. Bottom Right: obstacle is a safe distance away and the agent has resumed its idle state.

The programmatic flow of the obstacle avoidance module (Fig. 5) consists of (1) sweeping over each Lidar scan, computing the differences between successive radials to extract edges, (2) constructing a list of different regions, (3) determining if any identified obstacles pose a risk to the agent, and (4) generating the appropriate motor commands as a response. Instantiating a list of regions in this way creates the organizational framework for interacting with more complex behavioral modules reliant on feature extraction. 


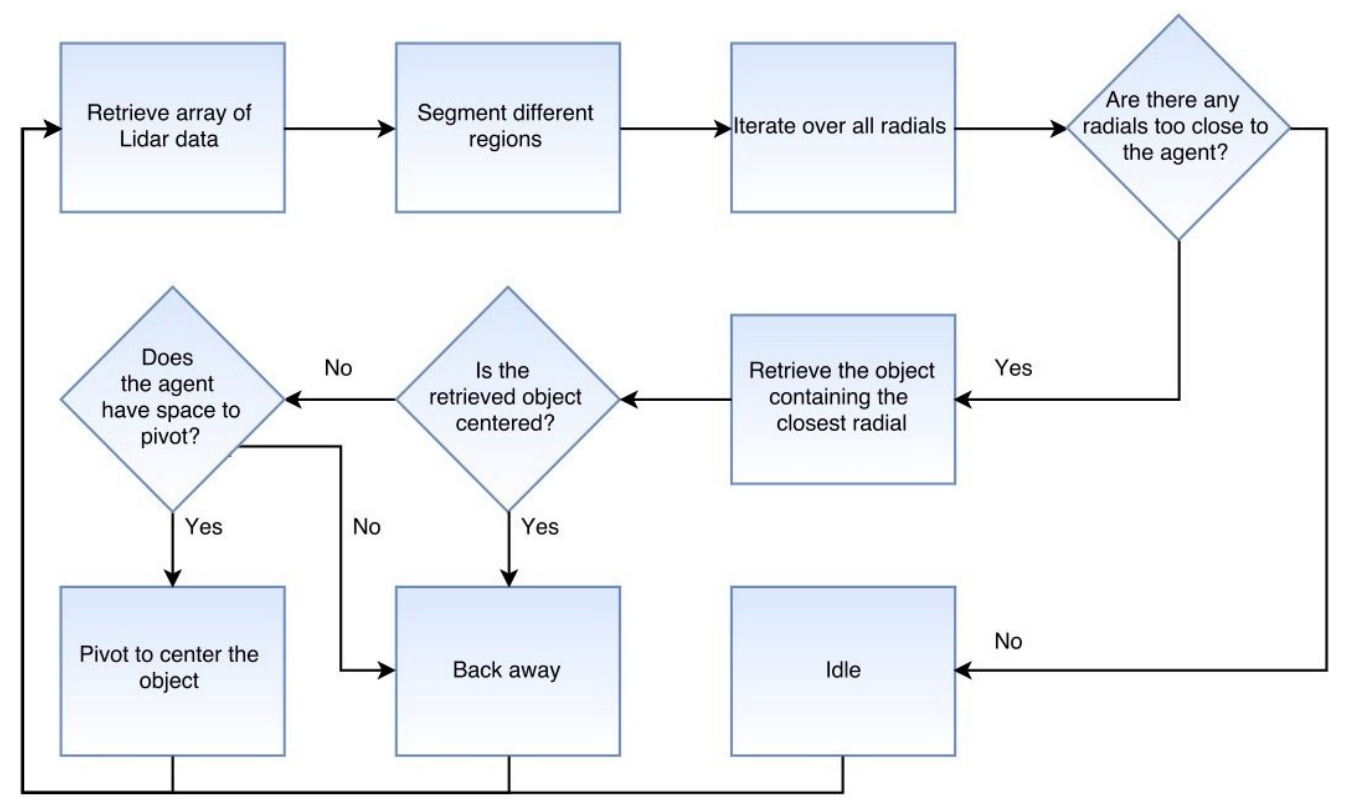

Figure 6. Programmatic representation of the obstacle avoidance algorithm.

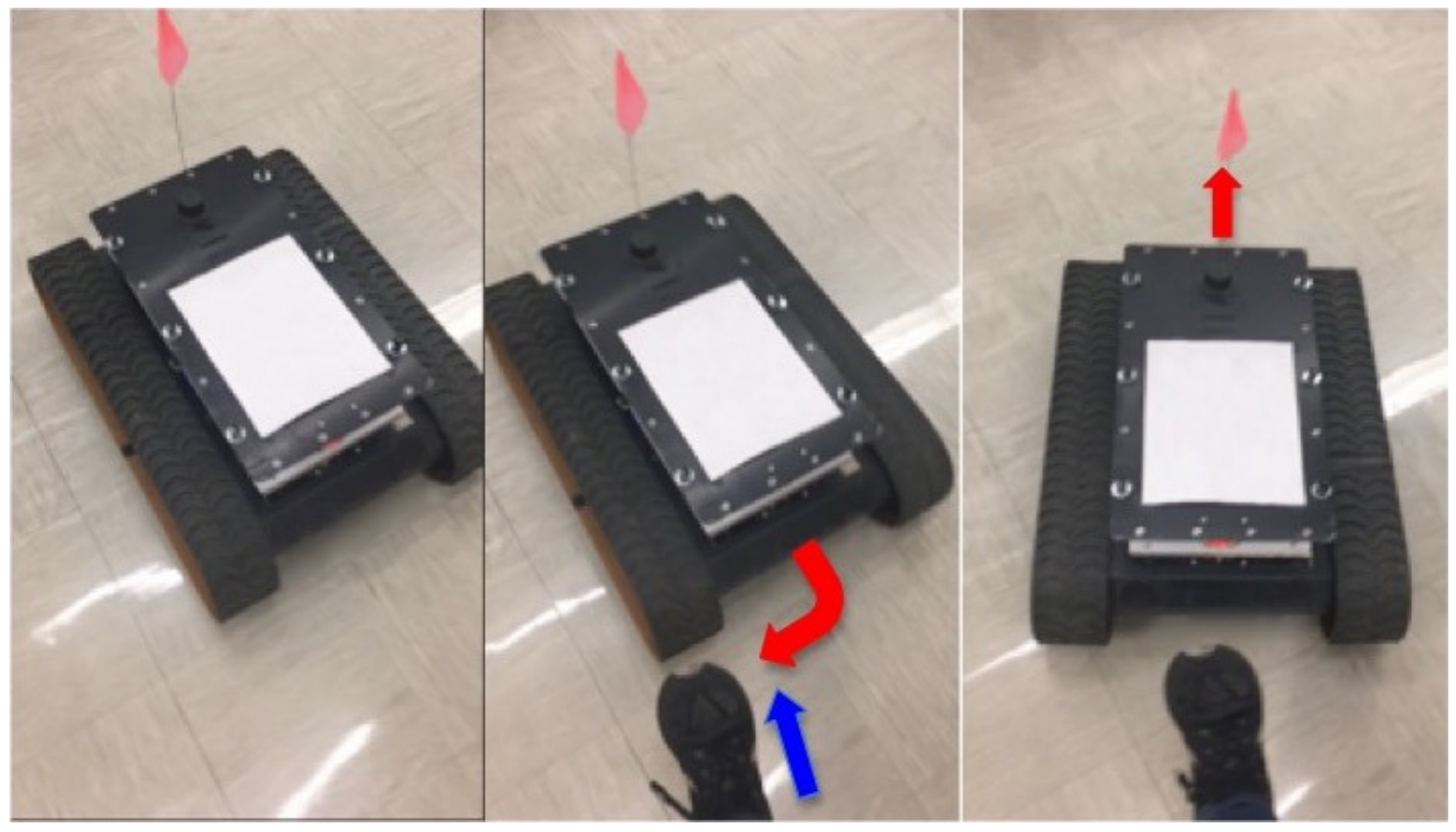

Figure 7. Implementation of the obstacle avoidance algorithm on a land rover. Left: rover is in idle state, as no obstacles are close enough to be a safety hazard. Middle: rover detects an obstacle and begins to center it via pivoting. Right: rover has completed its pivot and begins to back away from the obstacle.

\subsection{Constant Driving Motif}

The constant driving module builds on top of obstacle avoidance by adding a path-finding algorithm that (1) computes the average distance over each $10^{\circ}$ arc in the Lidar sweep, (2) checks if the deepest path is longer than a pre-defined minimum, and (3) responds by approaching the path, centering it, or turning around to find a new path as appropriate. The logical flow of the constant driving module is illustrated below (Fig. 8). 


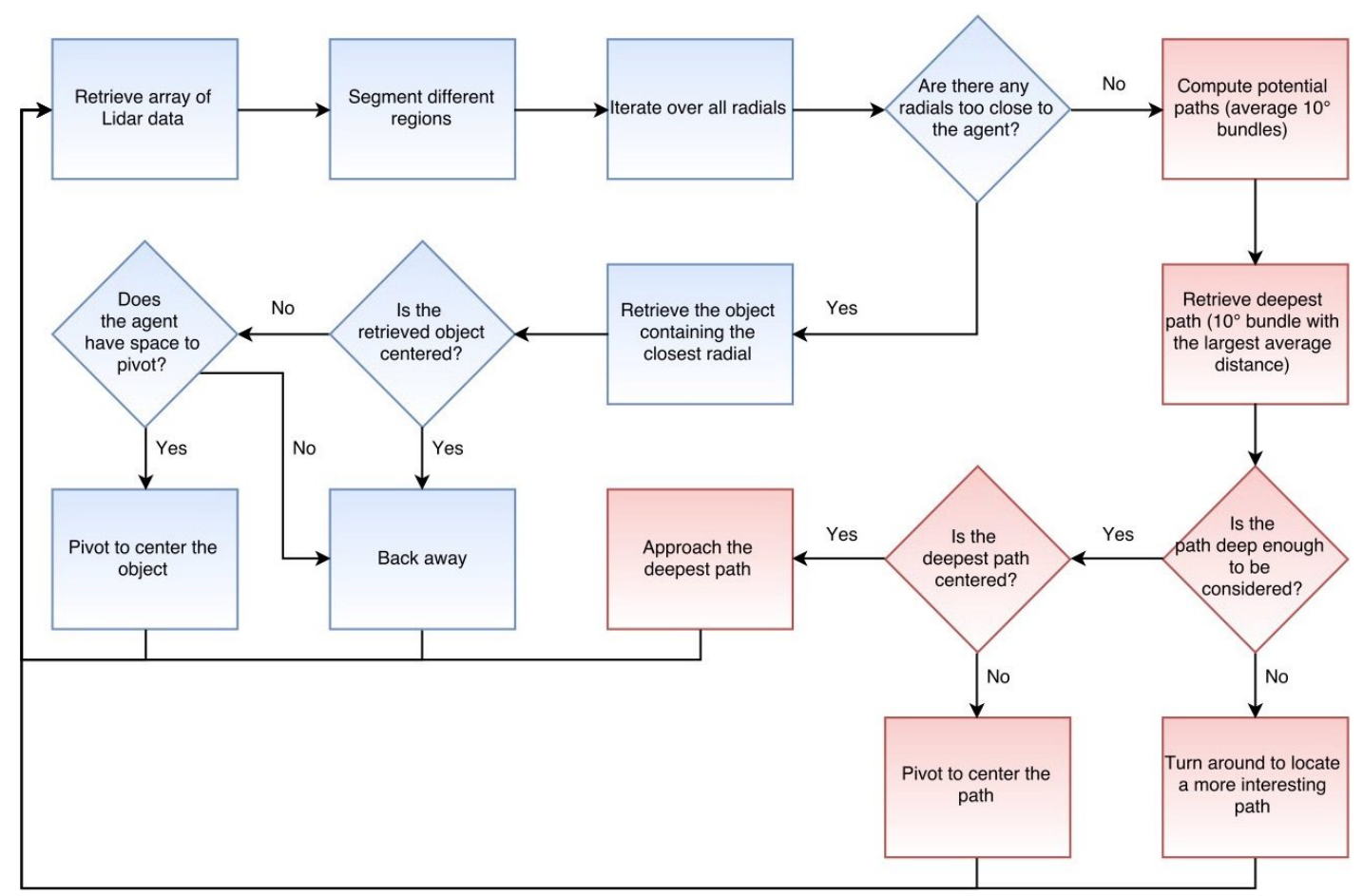

Figure 8. Programmatic representation of the constant driving algorithm. Blue: obstacle avoidance module. Red: expansions characterizing the primary behavior of the constant driving module.

Note that although the behavior characterizing the constant driving module replaces the idle state in the previously described obstacle avoidance motif (Section 3.1, Fig. 6), the internal structure of the obstacle avoidance core is otherwise identical to that described in Section 3.1. As a result, the obstacle avoidance and path-finding algorithm characterizing constant driving are largely isolated within the code. In the same way, the constant driving module will be used as a building block for more sophisticated behaviors such as object tracking and consequent navigational algorithms that minimize paths to specific destinations by going around obstacles.

\section{AUTOMATED GLOBAL FEATURE ANALYZER (AGFA): A DRIVER FOR TIER-SCALABLE AUTONOMY}

To address the above stated requirements for operational autonomy (Section 1) in a non-specific, generally applicable way, the lab has been developing the Caltech-patented Automated Global Feature Analyzer (AGFA), ${ }^{19-23}$ which performs automated target identification using image segmentation, feature extraction, classification, unbiased anomaly detection (i.e., based on feature space only), and target prioritization ${ }^{24}$ within mapped or imaged areas. AGFA enables automated science analysis aboard robotic spacecraft, and, embedded in tier-scalable reconnaissance mission architectures, is a primary driver of future intelligent, distributed, and autonomous robotic exploration.

AGFA objectively identifies and characterizes operational areas, both from an aerial/space perspective (i.e., overhead reconnaissance) and from the surface level. Moreover, it objectively flags anomalous targets via image processing, i.e., based on the sensor-provided feature space alone as opposed to user bias. The software's target identification and prioritization capabilities set the groundwork for autonomous surface investigation via robotic behavior modules/motifs (Section 3) in addition to robust path planning and adequate land/sea rover distribution in the presence of multiple targets and anomalies. 


\subsection{AGFA Classification Procedure}

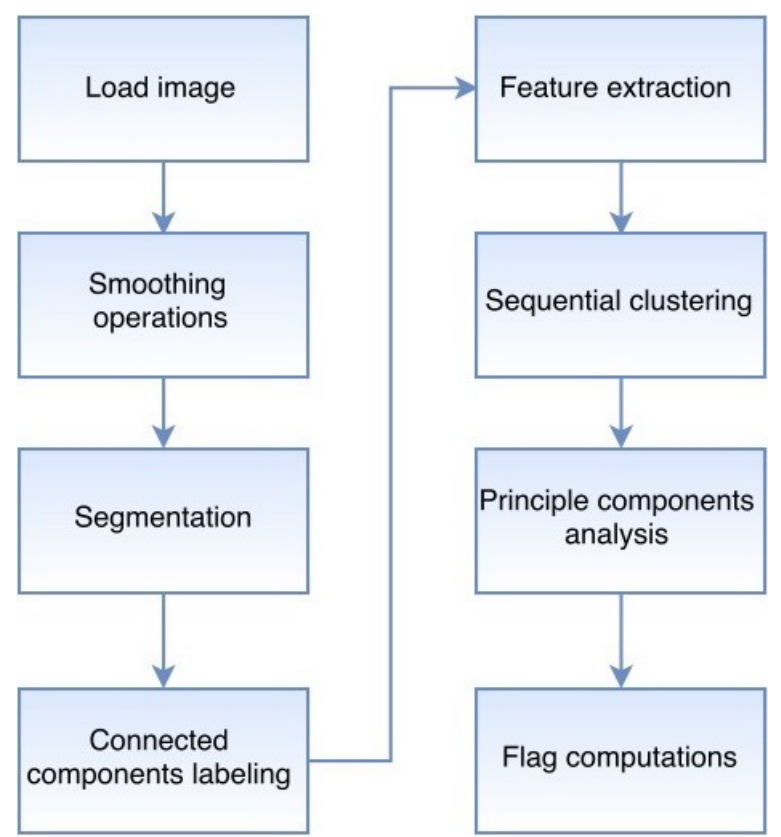

Figure 9. Logical flow of the Automated Global Feature Analyzer ${ }^{19-23}$ anomaly detection scheme.

AGFA's classification procedure (Fig. 9) consists of image smoothing via application of a mean-shift filter, ${ }^{25}$ image segmentation, ${ }^{26,27}$ connected components labeling, ${ }^{28}$ feature computations for each discovered/segmented object, ${ }^{21-23}$ sequential clustering, ${ }^{29}$ principal component analysis (PCA), ${ }^{30}$ and flag computations indicating the presence of relative and absolute anomalies for clusters as well as individual features. ${ }^{21-23}$

The features to be extracted and considered by the primary clustering algorithm are summarized below:

- $\quad$ Color - The average RGB components computed over an object surface.

- Albedo - The average brightness or shade of the object surface.

- Circularity: The pixel length ratio of the minor and major axes of the object's best-fitting ellipse.

- Angularity - The pixel length ratio of the best-fitting ellipse circumference and the object boundary.

- Compactness - The pixel length ratio of the best-fitting ellipse area and the object area.

- $\quad$ Moments - The object Alt and Hu moments.

- $\quad$ Texture - The object texture with respect to Gabor filters ${ }^{31-34}$ of varying scale and orientation.

Upon completion of feature-based clustering, the following flags are calculated to quantify anomalous targets:

- Distance Flag - The distance flag evaluates the similarity of clusters within the multi-dimensional feature space that is defined by the deployed sensors aboard the robotic agents within the TSR architecture. The largest eigenvalue of each cluster is extracted through principal component analysis (PCA). If the Euclidian distance in feature space between the centers of two clusters to be compared is larger than the sum of their respective maximum eigenvalues, the distance flag will be set to red, i.e., anomalous. Otherwise, it will be set to green, i.e., not anomalous.

- Number Flag - The number flag is used to evaluate population size ratios between clusters. If one cluster is significantly smaller than another it is compared against, the number flag will be set to red, i.e., anomalous. Otherwise, it will be set to green, i.e., not anomalous. Currently, we require the smaller cluster to be less than ten percent of the size of the larger cluster to warrant a red flag. Since this a user-defined parameter, future efforts will be targeted at removing this limitation to fully automate the number flag calculation. 


\subsection{AGFA Real World Application Example}

In the following we present an example of AGFA's feature extraction mechanisms at work on an unmodified image of water ice on Mars (Fig. 10) as seen by the Phoenix Mars lander. ${ }^{35}$ The images below (Fig. 11) illustrate different steps in AGFA's processing cycle, and are automatically generated as benchmarks upon completion of the image smoothing, connected components labeling, and feature extraction steps, respectively.

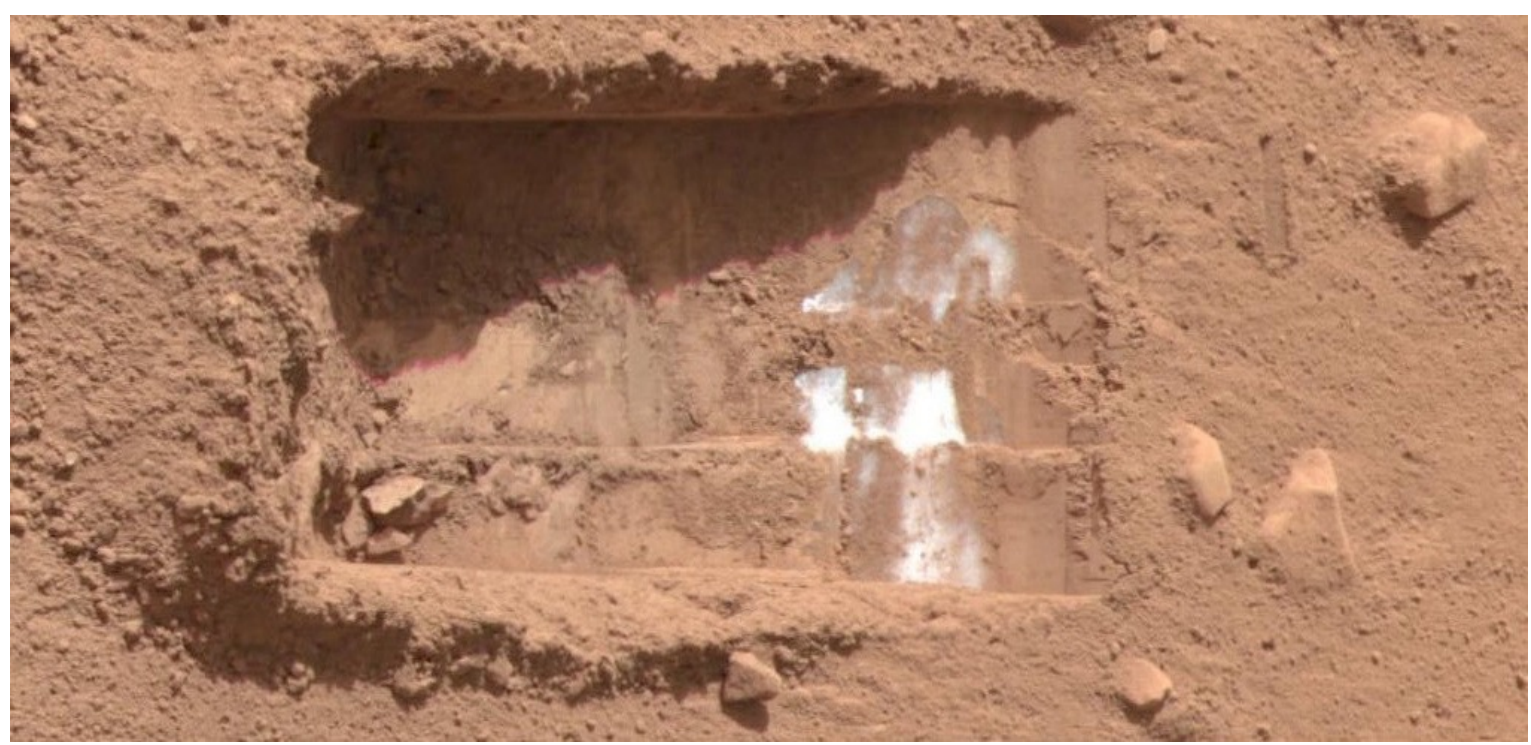

Figure 10. Unenhanced image (rotated by $90^{\circ}$ to the right) of water ice discovered in the Dodo-Goldilocks trench on Mars by NASA's Phoenix lander. [Image credit: NASA/JPL-Caltech/University of Arizona/Texas A\&M University]

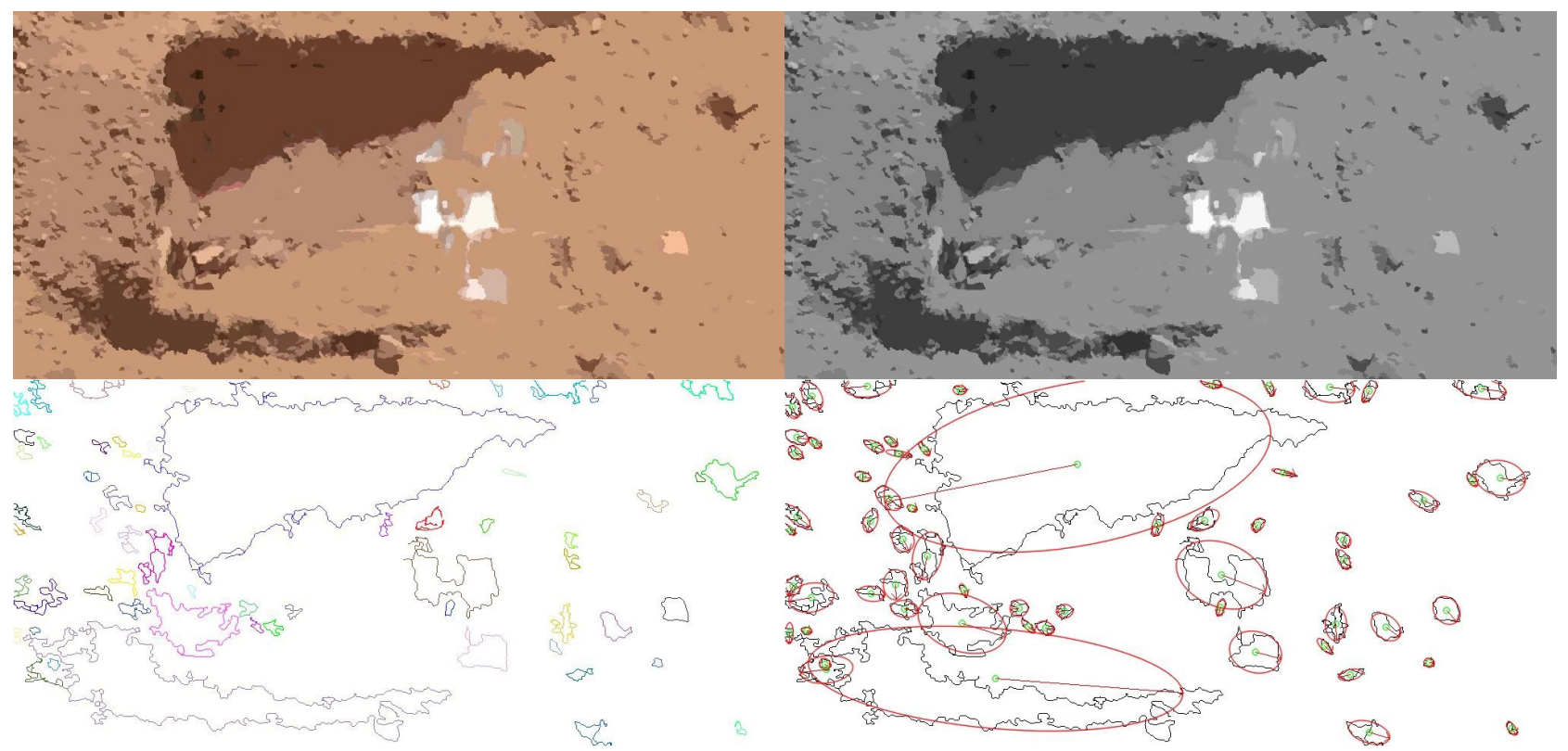

Figure 11. Top Left: result after applying mean shift filter for image smoothing. Top Right: grey-scale conversion of smoothed image. Bottom Left: connected components labeling after segmentation and pruning. Bottom Right: best-fitting ellipses for segmented objects (used for computing circularity, angularity, and compactness feature values).

AGFA's objective feature-based analysis automatically analyzes and generates four different clusters (indicated by four different colors, Fig. 12) of objects from the original image (Fig. 10). The blue and green clusters are of little interest and represent the average landscape in the image. The red cluster contains the shadows of the trench. The grey cluster encapsulates the vast majority of water ice regions, as well as a few rocks (due to similar ellipse fit characteristics). 


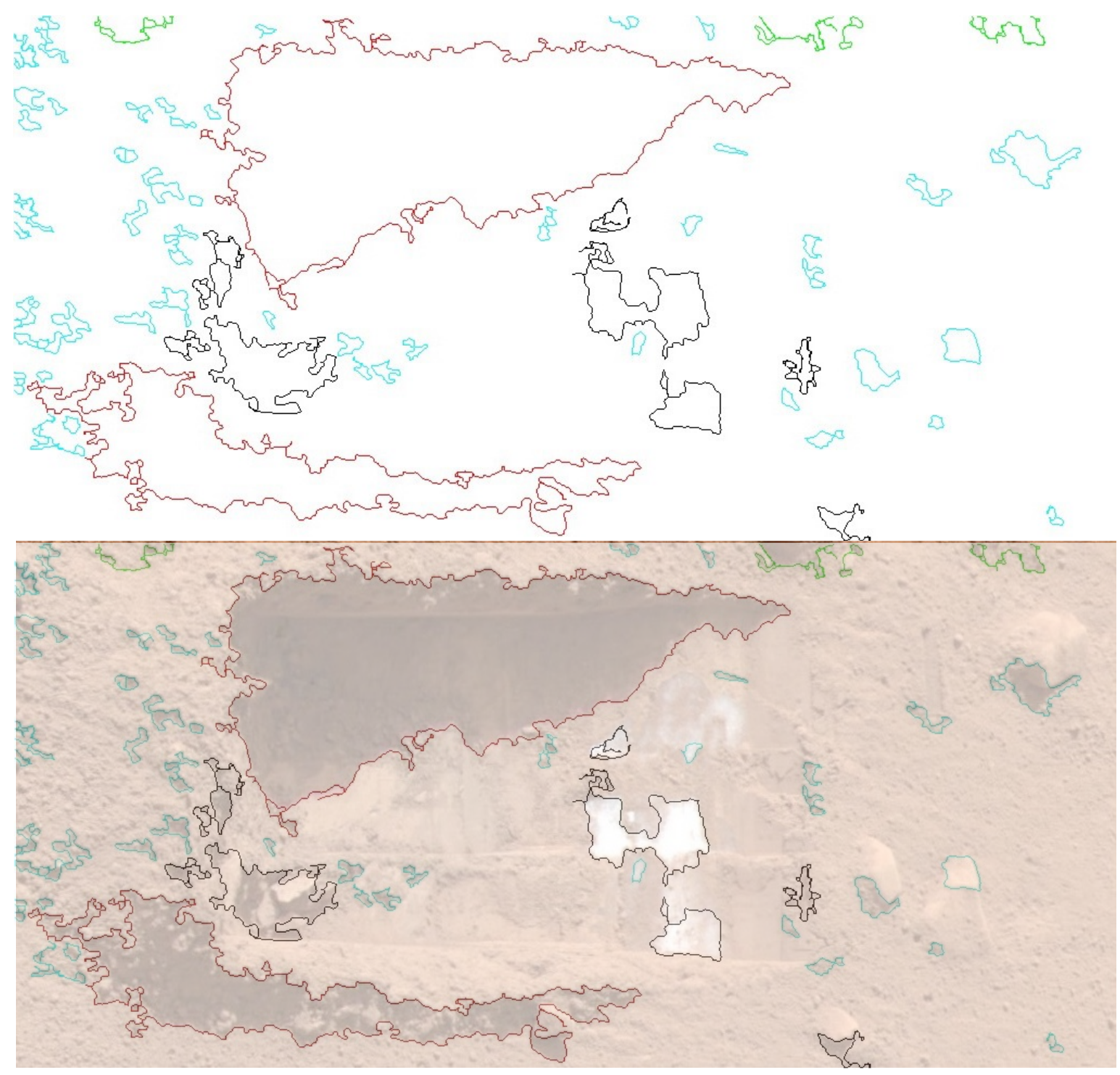

Figure 12. Top: color-map illustrating the four different clusters resulting from AGFA's feature-based sequential clustering. Bottom: color-map superimposed on original image (Fig. 10).

\begin{tabular}{|c|c|c|c|c|}
\hline Cluster & 1 (blue) & 2 (grey) & 3 (red) & 4 (green) \\
\hline 1 (blue) & & & & \\
\hline 2 (grey) & & & & \\
\hline 3 (red) & & & & \\
\hline 4 (green) & & & & \\
\hline
\end{tabular}

Figure 13. Upper (black) triangle: distance flag computed between clusters. Lower (white) triangle: number flag computed between clusters. 
Flag computations (Fig. 13) illustrate the anomalous characteristics of the cluster containing the water ice in terms of the distance flag. In contrast, the cluster containing the shadows is considered anomalous per the number flag because there are only two cluster members, i.e., two shadow regions.

Flag computations can also be extended to individual feature vectors (Fig. 14) by creating a difference matrix and applying an identical clustering algorithm. The resulting analysis indicates the presence of absolute anomalies in circularity, angularity, albedo, and compactness, as well as relative anomalies in color. Especially the absolute anomaly in the albedo feature is directly attributable to the presence of the white water ice patches.

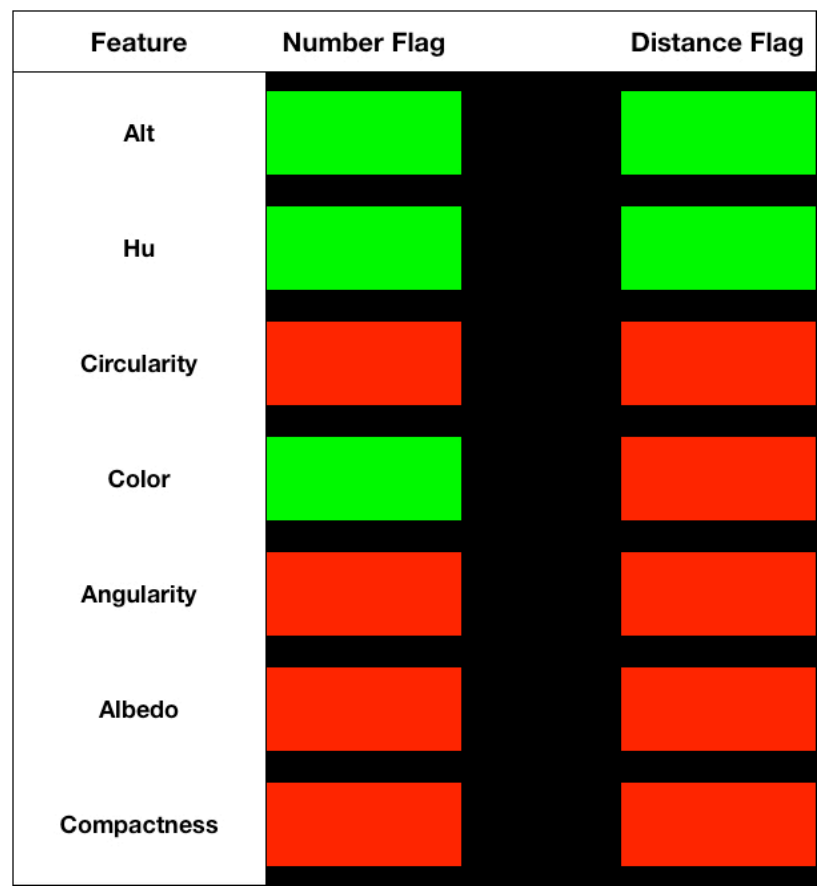

Figure 14. Individual feature classification flags computed based on sequential clustering with respect to normalized differences in feature space. Two green flags: no anomalies. One green flag and one red flag: relative anomalies detected. Two red flags: absolute anomalies detected.

\section{OUTLOOK - NEXT STEPS}

The next steps in the development of the TSR robotic outdoor testbed are the incorporation and integration of aerial platforms (air rovers, such as quadcopters and blimps or aerostats). The aerial tier will also serve as an emulation of a future space tier by deploying two aerial tiers: one being at a much higher altitude (i.e., space tier) than the other (i.e., aerial tier). Overall, the introduction of aerial tiers, i.e., overhead reconnaissance (Fig. 1), will necessitate the development of recognition algorithms to locate the surface agents (i.e., land and sea rovers) on the ground (see, e.g., [12]) for autonomous telecommanding via the overhead reconnaissance. The development of AGFA will continue with particular emphasis on strengthening the initial image segmentation step. Moreover, additional features will be added to AGFA, and a target prioritization framework, detailed in [24], will be merged with AGFA. Ultimately, the TSR robotic testbed will be deployed outdoors and subjected to and validated against various geological test scenarios.

\section{ACKNOWLEDGEMENTS}

The work described in this publication was carried out in part at the California Institute of Technology (2003 - 2016) and at the University of Arizona (2009 - present) with partial support from the Edward \& Maria Keonjian Endowment at the University of Arizona. JMD was partially supported by JSPS KAKENHI (Grant-in-Aid for Scientific Research on Innovative Areas) Grant Number 26106002 (Hadean BioScience). 
We are profoundly saddened by the passing of Larry Gilbert, Founding Director of the Office of Technology Transfer at the California Institute of Technology. We are deeply indebted to Larry, for his unwavering support of our Tier-Scalable Reconnaissance Paradigm as well as the associated Automated Global Feature Analyzer. This includes his never-ending thoughtful consideration of our long-term vision of TSR and its necessary utility in optimizing results which include (extraterrestrial) science return and national security. We sincerely hope that Larry will be remembered well into the future, as TSR continues to be applied to Earth and beyond.

\section{AUTHOR DISCLOSURE STATEMENT}

Authors WF, JMD, and MAT may have financial interest in the technologies presented here as they are named as inventors on several issued Caltech patents and pending University of Arizona patents on the underlying technologies. Author AJ-WB has no financial interest in the technologies presented.

\section{REFERENCES}

[1] Fink W, Dohm JM, Tarbell MA, Hare TM, Baker VR (2005) Next-Generation Robotic Planetary Reconnaissance Missions: A Paradigm Shift; Planetary and Space Science, 53, 1419-1426.

[2] Fink W, Dohm JM, Tarbell MA, Hare TM, Baker VR, Schulze-Makuch D, Furfaro R, Fairen AG, Ferre TPA, Miyamoto H, Komatsu G, Mahaney WC (2007) Tier-Scalable Reconnaissance Missions For The Autonomous Exploration Of Planetary Bodies; IEEE Aerospace Conference Proceedings, paper \#1199; DOI: 10.1109/AERO.2007.352715

[3] Noor AK, Cutts JA, Balint TS (2007) Platforms for discovery: Exploring Titan and Venus; Aerospace America/June 2007.

[4] Fink W, George T, Tarbell MA (2007) Tier-Scalable Reconnaissance: The Challenge of Sensor Optimization, Sensor Deployment, Sensor Fusion, and Sensor Interoperability; Proc. SPIE, Vol. 6556, 655611 (2007); DOI:10.1117/12.721486.

[5] Fink W, Tarbell MA, Jobling FM (2008) Tier-Scalable Reconnaissance - A Paradigm Shift in Autonomous Remote Planetary Exploration of Mars and Beyond; Chapter 1 in "Planet Mars Research Focus", Ed. L. A. Costas. Nova Science Publishers, Hauppauge, NY. ISBN: 1-60021-826-1.

[6] Kean S (2010) Making Smarter, Savvier Robots; Science, 329, 508-509.

[7] Fink W, Tarbell MA, Furfaro R, Powers L, Kargel JS, Baker VR, Lunine J (2011) Robotic Test Bed for Autonomous Surface Exploration of Titan, Mars, and Other Planetary Bodies; IEEE Aerospace Conference Proceedings, paper \#1770, Big Sky, Montana.

[8] Fink W, Tarbell MA (2007) Tier-scalable Reconnaissance Mission Test Bed: Implementation of Ground-Tier [abstract 2410]. In 38th Lunar and Planetary Science Conference Abstracts [CD-ROM], Lunar and Planetary Institute, Houston.

[9] Fink W, Tarbell MA (2009) Multi-Rover Test Bed for Tele-Conducted and Autonomous Surveillance, Reconnaissance, and Exploration; Proc. SPIE, Vol. 7331, 73310B (2009); DOI: 10.1117/12.819991

[10]Fink W, Tarbell MA (2009) CYCLOPS: A Mobile Robotic Platform for Testing and Validating Image Processing and Autonomous Navigation Algorithms in Support of Artificial Vision Prostheses; Comput Methods Programs Biomed, 96(3):226-33; DOI: 10.1016/j.cmpb.2009.06.009

[11]Fink W, Tarbell MA, Furfaro R, Powers L, Kargel JS, Baker VR, Lunine J (2011) Robotic Test Bed for Autonomous Surface Exploration of Titan, Mars, and Other Planetary Bodies; IEEE Aerospace Conference Proceedings, paper \#1770, Big Sky, Montana

[12]Fink W, Tuller M, Jacobs A, Kulkarni R, Tarbell MA, Furfaro R, Baker VR (2012) Robotic Lake Lander Test Bed for Autonomous Surface and Subsurface Exploration of Titan Lakes; IEEE Aerospace Conference Proceedings, paper \#1285, Big Sky, Montana

[13] Fink W, Baker VR, Schulze-Makuch D, Hamilton CW, Tarbell MA (2015) Autonomous Exploration of Planetary Lava Tubes Using a Multi-Rover Framework; IEEE Aerospace Conference Proceedings, paper \#2723, Big Sky, Montana 
[14]Fink W, Baker VR, Schulze-Makuch D, Hamilton CW, Tarbell MA (2015) Multi-Rover Framework to Autonomously Explore Planetary Lava Tubes [abstract 3011]. In 46th Lunar and Planetary Science Conference Abstracts [CD-ROM], Lunar and Planetary Institute, Houston

[15]Fink W, Tarbell M (2015) Autonomous C4ISR Systems for Extreme Environments; 2015 Defense Energy Innovation Summit, abstract and poster presentation

[16] US 6,990,406: "MULTI-AGENT AUTONOMOUS SYSTEM" (Fink, Dohm, Tarbell), assigned to Caltech

[17] US 7,734,063: "MULTI-AGENT AUTONOMOUS SYSTEM" (Fink, Dohm, Tarbell), assigned to Caltech

[18] US 7,742,845: "MULTI-AGENT AUTONOMOUS SYSTEM AND METHOD” (Fink, Dohm, Tarbell), assigned to Caltech

[19]Fink W, Datta A, Baker V (2005) AGFA: (Airborne) Automated Geologic Field Analyzer, Symposium SS-56: "New results from the robotic exploration of Mars and Titan and their implications on planetary environmental conditions and cosmochemistry". In Abstracts of the 15th Annual V.M. Goldschmidt Conference, Moscow, Idaho. Geochimica et Cosmochimica Acta, Volume 69, Number 10S, A535; abstract and poster

[20]Fink W, Datta A, Dohm JM, Tarbell MA, Jobling FM, Furfaro R, Kargel JS, Schulze-Makuch D, Lunine JI, Baker VR (2008) Automated Global Feature Analyzer (AGFA) for the Intelligent and Autonomous Robotic Exploration of the Solar System [abstract 1883]. In 39th Lunar and Planetary Science Conference Abstracts [CD-ROM], Lunar and Planetary Institute, Houston

[21] Fink W, Datta A, Dohm JM, Tarbell MA, Jobling FM, Furfaro R, Kargel JS, Schulze-Makuch D, Baker VR (2008) Automated Global Feature Analyzer (AGFA) - A Driver for Tier-Scalable Reconnaissance; IEEE Aerospace Conference Proceedings, paper \#1273; DOI: 10.1109/AERO.2008.4526422

[22] US 9,122,956 (and PCT): “Automated feature analysis, comparison, and anomaly detection" (Fink), assigned to Caltech

[23] US 9,424,489: "Automated feature analysis, comparison, and anomaly detection" (Fink), assigned to Caltech

[24] Fink W (2006) Generic Prioritization Framework for Target Selection and Instrument Usage for Reconnaissance Mission Autonomy, Proceedings of IEEE World Congress on Computational Intelligence (WCCI) 2006, Vancouver, Canada, 11116-11119

[25] Comaniciu D, Meer P (2002) Mean Shift: A Robust Approach Toward Feature Space Analysis; IEEE Transactions on Pattern Analysis and Machine Intelligence, Vol. 24, No. 5, MAY 2002603

[26] Otsu N (1979) A Threshold Selection Method from Gray-Level Histograms; IEEE Transactions on Systems, Man, and Cybernetics, Vol. SMC-9, No. 1, January 1979

[27] Canny J (1986) A Computational Approach to Edge Detection; IEEE Transactions on Pattern Analysis and Machine Intelligence, Vol. PAMI-8, No. 6, November 1986

[28] Russ JC (2002) The Image Processing Handbook, Fourth Edition, CRC Press, 2002

[29] Trahanias P, Skordalakis E (1989) An Efficient Sequential Clustering Method; Pattern Recognition, Vol. 22, No. 4 , pp 449-453, 1989

[30] Jolliffe IT (2002) Principal Component Analysis, Second Edition, Springer, 2002

[31] Gabor D (1946) Theory of communication; J IEE (London) 93:429-457

[32] Daugman JG (1980) Two-dimensional spectral analysis of cortical receptive field profiles; Vision Res., 20 (10): 847-56, doi:10.1016/0042-6989(80)90065-6

[33] Daugman JG (1987) Image analysis and compact coding by oriented 2-D Gabor primitives; SPIE Proc 758:19-30

[34]Fogel I, Sagi D (1989) Gabor filters as texture discriminator; Biological Cybernetics. 61 (2). doi:10.1007/BF00204594

[35] Arvidson RE, Bonitz RG, Robinson ML, Carsten JL, Volpe RA, Trebi-Ollennu A, Mellon MT, Chu PC, Davis KR, Wilson JJ, Shaw AS, Greenberger RN, Siebach KL, Stein TC, Cull SC, Goetz W, Morris RV, Ming DW, Keller HU, Lemmon MT, Sizemore HG, Mehta M (2009) Results from the Mars Phoenix Lander Robotic Arm experiment; Journal of Geophysical Research, Vol. 114, E00E02, doi:10.1029/2009JE003408, 2009 